\title{
Synthesis of Biomaterial-Based Hydrogels Reinforced with Cellulose Nanocrystals for Biomedical Applications
}

\author{
Pavan Kumar Dara $\mathbb{D}^{1},{ }^{1}$ Mahadevan Raghavankutty $\mathbb{D}^{1},{ }^{1}$ Karthik Deekonda $\mathbb{D},{ }^{2}$ \\ Anil Kumar Vemu $\mathbb{D}^{3}$, Visnuvinayagam Sivam ${ }^{(D)}{ }^{4}$ Suseela Mathew ${ }^{(D)}{ }^{1}$ \\ Anandan Rangasamy $\mathbb{D}^{1},{ }^{1}$ Ravishankar Chandragiri Nagarajarao $\mathbb{D D}^{1}$ \\ and Senthilkumar Subramanian ${ }^{5}$ \\ ${ }^{1}$ Biochemistry and Nutrition Division, ICAR-Central Institute of Fisheries Technology, Cochin, 682029 Kerala, India \\ ${ }^{2}$-Chimertech Innovations LLP, Veterinary Incubation Foundation @ TANUVAS, CUL Building, Madhavaram Milk Colony, \\ Chennai, 600051 Tamil Nadu, India \\ ${ }^{3}$ Department of Microbiology, Government Medical College, Ongole, 523001 Andhra Pradesh, India \\ ${ }^{4}$ Microbiology and Biotechnology, ICAR-Central Institute of Fisheries Technology, Cochin, 682029 Kerala, India \\ ${ }^{5}$ College of Medicine and Health Science, Jigjiga University, Ethiopia
}

Correspondence should be addressed to Senthilkumar Subramanian; senthilkumarsubramanian@jju.edu.et

Received 21 June 2021; Accepted 17 August 2021; Published 8 September 2021

Academic Editor: Senthilkumar Rajagopal

Copyright (c) 2021 Pavan Kumar Dara et al. This is an open access article distributed under the Creative Commons Attribution License, which permits unrestricted use, distribution, and reproduction in any medium, provided the original work is properly cited.

\begin{abstract}
Cellulose nanocrystals (CNC) were prepared by formic acid hydrolysis and TEMPO- (2,2,6,6-tetramethyl-piperidine-1-oxyl-) mediated oxidation. The prepared CNCs were reinforced into biopolymers chitosan (CHI), alginate (ALG), and gelatin (GEL) to obtain "CNC-ALG-GEL" and "CNC-CHI-GEL" hydrogels. The synthesized hydrogels were characterized for physicochemical, thermal, and structural characterization using Fourier transform infrared spectroscopy (FT-IR), scanning electron microscopy (SEM), thermal gravity analysis (TGA), and X-ray diffraction (XRD) analyses. Notably, the reinforcement of CNC has not altered the molecular structure of a biopolymer as revealed by FT-IR analysis. The hydrogels reinforced with CNC have shown better thermal stability and miscibility as revealed by thermal gravity analysis. The physicochemical, thermal, and structural characterization revealed the chemical interaction and electrostatic attraction between the CNC and biopolymers. The biocompatibility was investigated by evaluating the viability of the L929 fibroblast cell, which represents good biocompatibility and nontoxic nature. These hydrogels could be implemented in therapeutic biomedical research and regenerative medicinal applications.
\end{abstract}

\section{Introduction}

Cellulose is one of the naturally occurring renewable biopolymers. It plays a key position as an abundant raw organic material which is capable of meeting the demands for green and biobased products [1]. Nanocellulose materials such as cellulose nanocrystals (CNC) and cellulose nanofibrils (CNF) prepared from cellulose have gained tremendous attention in tissue engineering, biomedical applications, and regenerative medicine due to their superior biocompat- ibility and favourable rheological and biological active properties [2].

CNCs are rod-shaped-like nanoparticles that can be obtained from different types of diversified rich cellulose bioresources such as rice husk, wheat straw, cotton, and wood pulp [3]. It possesses a diameter of $2-20 \mathrm{~nm}$ with a length of 100-500 $\mathrm{nm}$. Generally, CNC can be extracted from cellulose biomaterial through acid hydrolysis using acids such as sulfuric acid, formic acid, hydrochloric acid, nitric acid, and phosphoric acid. Nevertheless, among all the acids, 
formic acid is mostly preferable due to its certain desirable abilities like being less corrosive, reusable, and easily recoverable and having a low boiling point [4]. Due to minimal cytotoxicity and immunogenicity properties, CNCs have the ability to be utilised as an extracellular matrix in tissue culture medium which enhances cell growth and cell proliferation [5].The immense capacity of CNC for surface charge modification allows researchers to improve their hydrophilicity and stability and thereby enhance the bioavailability of CNC [6]. The nanocrystals with high carboxyl content have been found to be suitable for drug delivery applications [7].

Over the past years, many researchers have attempted to develop extraction methods for the preparation of CNC. Many researchers have developed biopolymer-based materials such as hydrogels and scaffolds for wound dressing, biomedical applications, and therapeutic research. Several research studies have been extensively made for the incorporation/fabrication/injection of CNC into biopolymers such as alginate, chitosan, gelatin, polyvinyl alcohol, and hydroxyapatite [2, 5, 8-11]. Jeddi and Mahkam synthesized nanocarboxymethyl cellulose from CNC to incorporate in bilayer alginate-chitosan hydrogel beads and successfully use as a carrier for the controlled delivery of dexamethasone [12]. In another study, Ntoutoume et al. demonstrated that the curcumin and cyclodextrin complexes with $\mathrm{CNC}$ have an antiproliferative effect on colorectal and prostatic cancer cell lines [13]. Further, according to Bertsch et al., the presence of CNC in the polymer complex enhanced the physiological and rheological characteristics of the wound dressing material [14]. Due to the outstanding mechanical properties of CNC, innovative research could evolve for the development of artificial organs such as blood vessels, nuclear pulposus, and soft tissues by the application of $\mathrm{CNC}$ [15]. With this rationale, the aim of the present research investigation was to synthesize the CNC from cellulose by formic acid hydrolysis and reinforcement of synthesized CNC into the biopolymers chitosan, alginate, and gelatin for the preparation of hydrogels. The physicochemical, structural rheological, and cytotoxicity properties were assessed that play an important role in biomedical and tissue engineering applications.

\section{Materials and Methods}

Low molecular weight chitosan (degree of deacetylation with $75-85 \%, 50-190 \mathrm{kDa}$ ), sodium alginate, ferric (III) chloride $\left(\mathrm{FeCl}_{3}\right)$, formic acid, TEMPO (2,2,6,6-tetramethylpiperidine-1-oxyl), EDC (1-ethyl-3-(3-dimethylaminopropyl)carbodiimide), and sodium bromide $(\mathrm{NaBr})$ were purchased from Sigma, St. Louis, USA. All other chemicals and reagents used in the present study were of either analytical grade (AR) or guaranteed grade (GR).

2.1. Preparation of Cellulose Nanocrystals. CNC was prepared by formic acid hydrolysis according to the following method as described by Du et al. [4]. Briefly, $3 \mathrm{gm}$ of cellulose was added in $100 \mathrm{ml}$ of $0.015 \mathrm{M} \mathrm{FeCl}_{3}$ containing $90 \mathrm{ml}$ formic acid in a spherical flask. The flask was kept at $95^{\circ} \mathrm{C}$ for $4 \mathrm{~h}$ under stirring using a magnetic stirrer (Heidolph Instruments, GmbH and Co., Germany). The flask was cooled down to room temperature followed by centrifugation at $8000 \mathrm{rpm}$ for $10 \mathrm{~min}$ using centrifuge (Sorvall Legend XTR, Thermo Fisher Scientific, New Hampshire, USA). The obtained white residue was washed repeatedly with distilled water until pH 6 was achieved. After separation of the supernatant, the white residue nanocrystals were freeze dried at $-40^{\circ} \mathrm{C}$ for $72 \mathrm{~h}$ using a freeze dryer (Lark, Tamil Nadu, India) and stored at $4^{\circ} \mathrm{C}$ until further analysis.

The obtained white residue nanocrystals were subjected to cationic modification using TEMPO and sodium hypochlorite $(\mathrm{NaOCl})$ according to the following method as described by Akhlaghi et al. [6]. $1.5 \mathrm{gm}$ of nanocrystals was added to $100 \mathrm{ml}$ of deionised water and sonicated for $15 \mathrm{~min} .20 \mathrm{mg}$ of TEMPO and $400 \mathrm{mg}$ of $\mathrm{NaBr}$ were added to the $\mathrm{CNC}$ suspension. The $\mathrm{pH}$ of the $\mathrm{CNC}$ suspension was adjusted to 10 using $1 \mathrm{M} \mathrm{NaOH}$. The oxidation reaction was initiated by adding $10 \mathrm{ml}$ of $13 \% \mathrm{NaOCl}$ to the CNC suspension under gentle stirring continuously for $3 \mathrm{~h}$, simultaneously maintaining constant $\mathrm{pH} 10$. An aliquot of $10 \mathrm{ml}$ of methanol was added to quench the reaction, and $\mathrm{pH}$ was adjusted to 7 using $1 \mathrm{M} \mathrm{HCl}$. Finally, the oxidised nanocrystals were dialysed against deionised water using $14 \mathrm{kDa}$ dialysis membranes (Sigma, St. Louis, USA). The resultant freeze-dried cellulose nanocrystals were designated as "CNC."

2.2. Preparation of Hydrogels Reinforced with CNC. The biomaterial-based hydrogels reinforced with CNC were prepared according to the following method described by Wang et al. [16], with slight modifications. A $1 \%$ CNC $(20 \mathrm{ml})$ was dispersed in $40 \mathrm{ml}$ of $2 \%$ alginate and $2 \%$ chitosan homogenous solutions separately via sonication for $15 \mathrm{~min}$ and designated as "CNC-ALG" and "CNC-CHI," respectively. Further, a $2 \%$ gelatin was dissolved in $40 \mathrm{ml}$ of deionised water with $20 \mathrm{mg}$ of EDC (1-ethyl-3-(3-dimethylaminopropyl)carbodiimide) and stirred continuously for $24 \mathrm{~h}$ at room temperature. Fish gelatin was used in the present study, which was prepared according to the following method as described by Dara et al. [17]. This gelatin solution with EDC was added to CNC-ALG and CNC-CHI solutions followed by the addition of $0.5 \mathrm{ml}$ of $50 \mathrm{mM} \mathrm{ZnSO}_{4}$. The suspension was stirred continuously for $6 \mathrm{~h}$ at room temperature for ionic cross-linking. Finally, the obtained hydrogels were rinsed with $1 \mathrm{x}$ phosphate buffer saline (PBS) and designated as "CNC-ALG-GEL" and "CNC-CHI-GEL," respectively. The hydrogels were stored at $4^{\circ} \mathrm{C}$ until further analysis. The procedure for the preparation of hydrogels is shown in Figure 1.

\subsection{Particle Size Distribution and Zeta Potential Analysis.} The particle size distribution (PSD) and zeta potential of $\mathrm{CNC}$ and hydrogel samples were determined by dynamic light scattering analyses (DLS) using a Zetasizer Nano Series (Malvern, Worcestershire, UK). For PSD analysis, the CNC and hydrogel samples were loaded into a quartz cuvette with $1 \mathrm{~cm}$ path-length and were subjected to DLS measurement with a detection angle of $90^{\circ}$ at $25 \pm 0.1^{\circ} \mathrm{C}$. The $Z$-average 


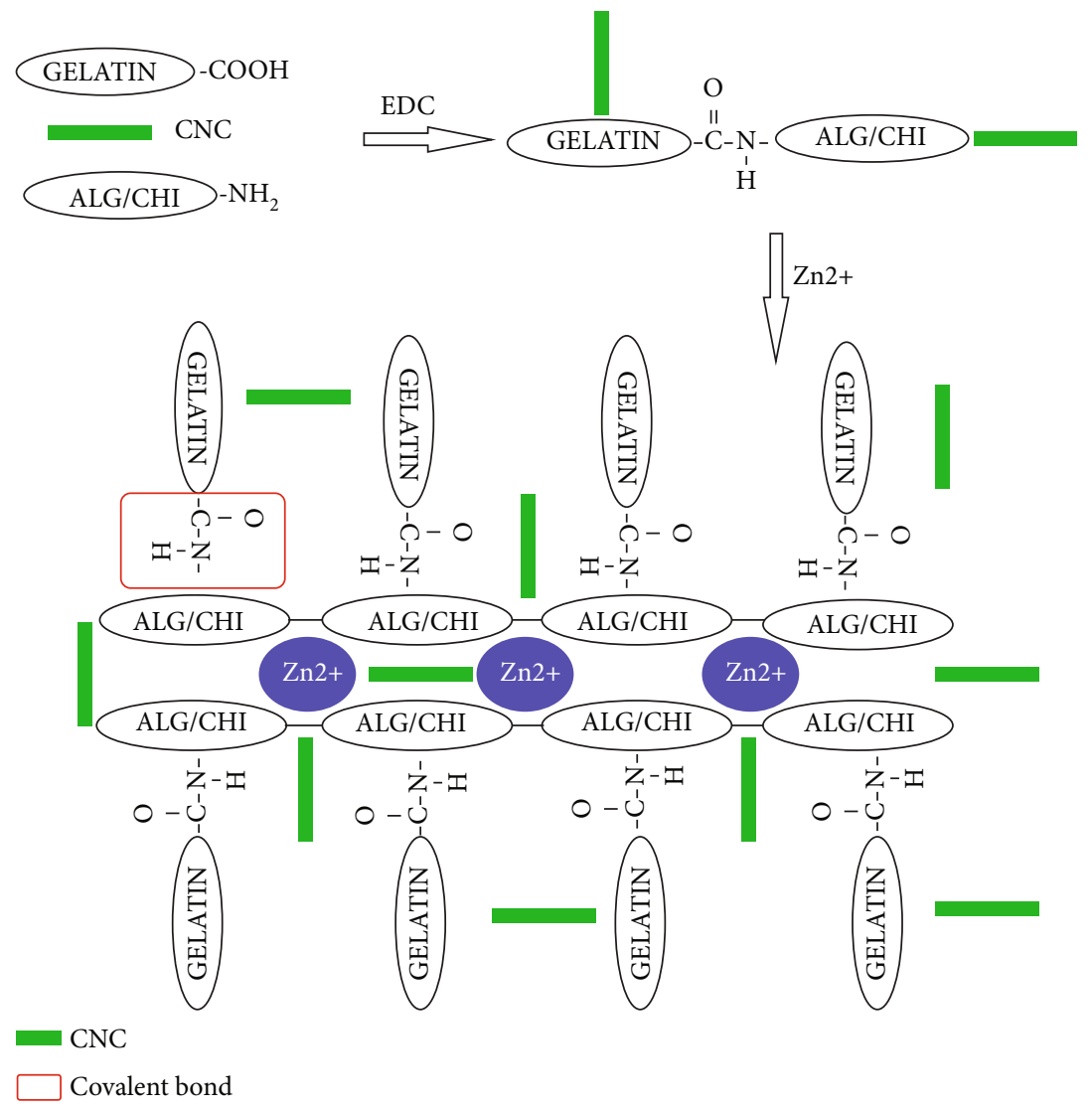

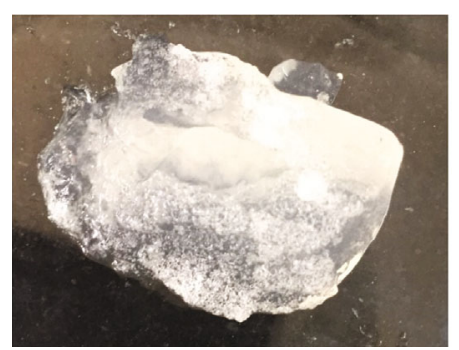

CNC-ALG-GEL

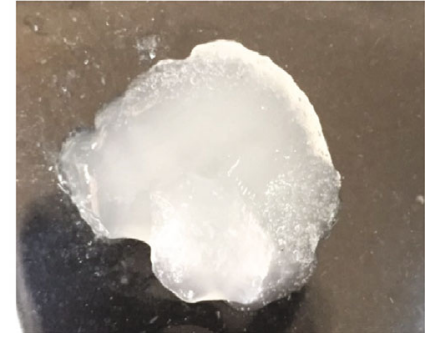

CNC-CHI-GEL

FIGURE 1: Procedure for the preparation of hydrogels reinforced with CNC.

hydrodynamic diameters and polydispersity index (PDI) of hydrogels were determined and recorded based on the scattering intensity. For analysing the zeta potential, the $\mathrm{CNC}$ and hydrogel samples were filtered using a $0.45 \mu \mathrm{m}$ syringe filter membrane to remove large particles and measured with an angle of $15^{\circ}$ at $25^{\circ} \mathrm{C}$.

2.4. FT-IR Spectra and SEM Morphology. The FT-IR spectra of CNC and hydrogels were analysed using a FT-IR spectrometer (Bruker Optik $\mathrm{GmBH}$, Germany), in the region of $4000 \mathrm{~cm}^{-1}-500 \mathrm{~cm}^{-1}$ wave numbers at $4 \mathrm{~cm}^{-1}$ resolution. The surface morphology was examined using scanning electron microscopy (SEM JSM, Japan), and samples were observed at an accelerated $15 \mathrm{kV}$ voltage.

2.5. X-Ray Diffraction (XRD) Pattern. X-ray powder diffraction patterns of CNC and hydrogels were recorded using an
Analytical X'Pert Pro X-ray diffractometer. The X-ray source was $\mathrm{Cu}-\mathrm{K} \alpha$ radiation $(40 \mathrm{kV}, 80 \mathrm{~mA})$. The XRD patterns of samples were taken in the $2 \theta$ range of $10^{\circ}-80^{\circ}$ in a fixed time mode at room temperature at a scanning rate of $4^{\circ} \mathrm{min}^{-1}$.

2.6. Thermal Characteristics. Prior to the analyses, the CNC and hydrogel samples were conditioned at $25^{\circ} \mathrm{C}$ and $53 \%$ relative humidity. DTG (derivative thermogravimetry) and DTA (differential thermal analysis) of hydrogels were analysed using a thermogravimetric analyser (Seiko Exstar 6300, Italy). Samples were heated from $600^{\circ} \mathrm{C}$, at $20^{\circ} \mathrm{C} / \mathrm{min}$ heating rate, using nitrogen flow $(250 \mathrm{ml} / \mathrm{min})$. The $T_{\mathrm{o}}$ (onset temperature), $T_{\mathrm{m}}$ (melting temperature), and $\Delta H$ (enthalpy) of hydrogel samples were obtained from DTG/DTA curves.

2.7. Rheological Study. The rheological flow properties (shear stress and viscosity vs. shear rate) of CNC and hydrogels 
were measured using a Brookfield DV-III Ultra ${ }^{\mathrm{TM}}$ Programmable Rheometer (Brookfield Engineering Laboratories Inc., Middleboro, USA). The cone and plate spindle (CP41 model) with measuring geometry of $5 \mathrm{~cm}$ was used with a gap of $0.05 \mathrm{~mm}$ and a shear rate ranging from 10 to $100 \mathrm{~s}^{-1}$. A flow curve was obtained by plotting shear stress and viscosity vs. shear rate values using a steady state flow program. The Herschel-Bulkley model was selected as the best-fit model based on standard error of shear stress-shear rate and shear stress-viscosity data.

The Herschel-Bulkley model equation is as follows:

$$
T=T_{\mathrm{o}}+k D^{\eta}
$$

where $T$ is the shear stress $(\mathrm{Pa}), T_{\mathrm{o}}$ is the yield stress $(\mathrm{Pa}), D$ is the shear rate $\left(\mathrm{s}^{-1}\right), k$ is the consistency coefficient, and $\eta$ is the flow behaviour index (dimensionless). The consistency coefficient $(k)$ and flow behaviour index $(\eta)$ of CNC and hydrogels were determined by the steady state flow program software.

2.8. In Vitro Degradation. In vitro degradation of CNC and hydrogels was studied using the enzymatic digestion method. A known weight of the hydrogel sample was taken and placed in an aliquot $(10 \mathrm{ml})$ of PBS with $10 \mathrm{U} / \mathrm{ml}$ of trypsin at $37^{\circ} \mathrm{C}$ for the duration of 7 days. The weight of hydrogel samples was taken periodically.

2.9. Cytotoxicity. Cytotoxicity/biocompatibility of CNC and hydrogels was investigated by MTT (3-(4,5-dimethylthiazol-2-yl)-2,5-diphenyltetrazolium bromide) assay according to the method followed Dara et al. [18]. The viability of cells was calculated and was expressed in percentage (\%).

$$
\text { Cell viablity }(\%)=\frac{\text { OD of sample }}{\text { OD of control }} \times 100
$$

2.10. Statistical Analysis. One-way ANOVA was used to analyse the data. Experiments were carried out in triplicates independently. Significant differences between the means of triplicates were determined by Duncan's multiple comparison test using statistical software IBM SPSS 20 (SPSS Inc., Illinois, USA).

\section{Results and Discussion}

3.1. Particle Size Distribution and Zeta Potential Analysis. Particle size distribution is one of the most important characteristic properties that determine its functionality, whereas the zeta potential defines the potential difference between the dispersion medium and the layer of fluid attached to the dispersed nanocrystals in a colloidal system of charged particles [10]. The $Z$-average hydrodynamic diameter, polydispersity index (PDI), and zeta potential data of hydrogels are displayed in Table 1 . The $Z$-average hydrodynamic diameter and zeta potential of synthesized CNC were found to be $192 \mathrm{~nm}$ and $-48.40 \mathrm{mV}$, respectively. The particle size of the CNC depends on several factors such as type of acid used for the hydrolysis, reaction temperature, and reaction
TABle 1: Particle size distribution and zeta potential of hydrogels.

\begin{tabular}{lccc}
\hline Samples & $\begin{array}{c}\text { Particle size } \\
(\mathrm{d} \cdot \mathrm{nm})\end{array}$ & PDI & $\begin{array}{c}\text { Zeta potential } \\
(\mathrm{mV})\end{array}$ \\
\hline CNC & $192 \pm 6.82$ & $0.275 \pm 0.0013$ & $-48.4 \pm 0.62$ \\
ALG-GEL & $251 \pm 7.80$ & $0.376 \pm 0.0110$ & $-24.4 \pm 2.06$ \\
CHI-GEL & $308 \pm 9.87$ & $0.405 \pm 0.0236$ & $61.5 \pm 5.87$ \\
CNC-ALG- & $415 \pm 14.30$ & $0.464 \pm 0.0215$ & $-61.6 \pm 7.89$ \\
GEL & & & \\
CNC-CHI- & $572 \pm 13.74$ & $0.488 \pm 0.0255$ & $16.9 \pm 1.96$ \\
GEL & & & \\
\hline
\end{tabular}

time [1]. In the present study, formic acid was used for the hydrolysis, and the reaction time and temperature were $4 \mathrm{~h}$ and $95^{\circ} \mathrm{C}$, respectively. The smaller particle size and higher zeta potential of synthesized CNC were most likely due to the improved dispersibility and conversion of hydroxyl groups to carboxyl groups on the biopolymer cellulose surface during the TEMPO-mediated oxidation process [19]. The $Z$-average hydrodynamic diameter and zeta potential of hydrogels CNC-ALG-GEL and CNC-CHI-GEL were found to be $415 \mathrm{~nm}$ and $572 \mathrm{~nm}$ and $-61.6 \mathrm{mV}$ and $+16.9 \mathrm{mV}$, respectively. It can be noted that the changes in the particle size and zeta potential of CNC-ALG-GEL and CNC-CHI-GEL represent the colloidal electrostatic interactions between the incorporated $\mathrm{CNC}$, chitosan, and alginate biopolymers. The negative zeta potential value of CNCALG-GEL $(-61.6 \mathrm{mV})$ indicates the presence of carboxyl groups, and the positive value of CNC-CHI-GEL confers the cationic characteristics of chitosan chains $[18,20]$. Further, the lowest zeta potential $(+16.9 \mathrm{mV})$ of CNC-CHIGEL might be due to the neutralization of amino groups on biopolymer chitosan that results from the separation from CNC and aggregation at the surface of nanocrystals [21]. Nevertheless, the nature of the biopolymers used for the surface modification defines the surface charge. In the present study, the positive zeta potential of CNC-CHI-GEL and CHI-GEL represents the cationic characteristic of chitosan, whereas the negative zeta potential of CNC-ALG-GEL and ALG-GEL could be originated from the carboxylic groups of alginate. The smaller particle size of CNC-ALGGEL might be due to more affinity and adsorption of the cellulose nanocrystal to the surface of the biopolymer. Moreover, the swelling of cellulose nanocrystals tends to create repulsion between polymeric chains which results in increasing the particle size [15]. It can be noticed that the synthesized CNC and hydrogels were monodispersed which was evident by the low PDI $(<0.5)$. Furthermore, the detailed morphological studies of $\mathrm{CNC}$ and hydrogels were confirmed by SEM. From this data, it can be deduced that the biopolymer hydrogels were highly stable due to reinforcement of CNC.

3.2. FT-IR Spectra. FT-IR spectral analysis was performed to study the alterations in the functional groups of the hydrogels that would have occurred due to cross-linking of CNC. The FT-IR spectra of CNC were found to have 


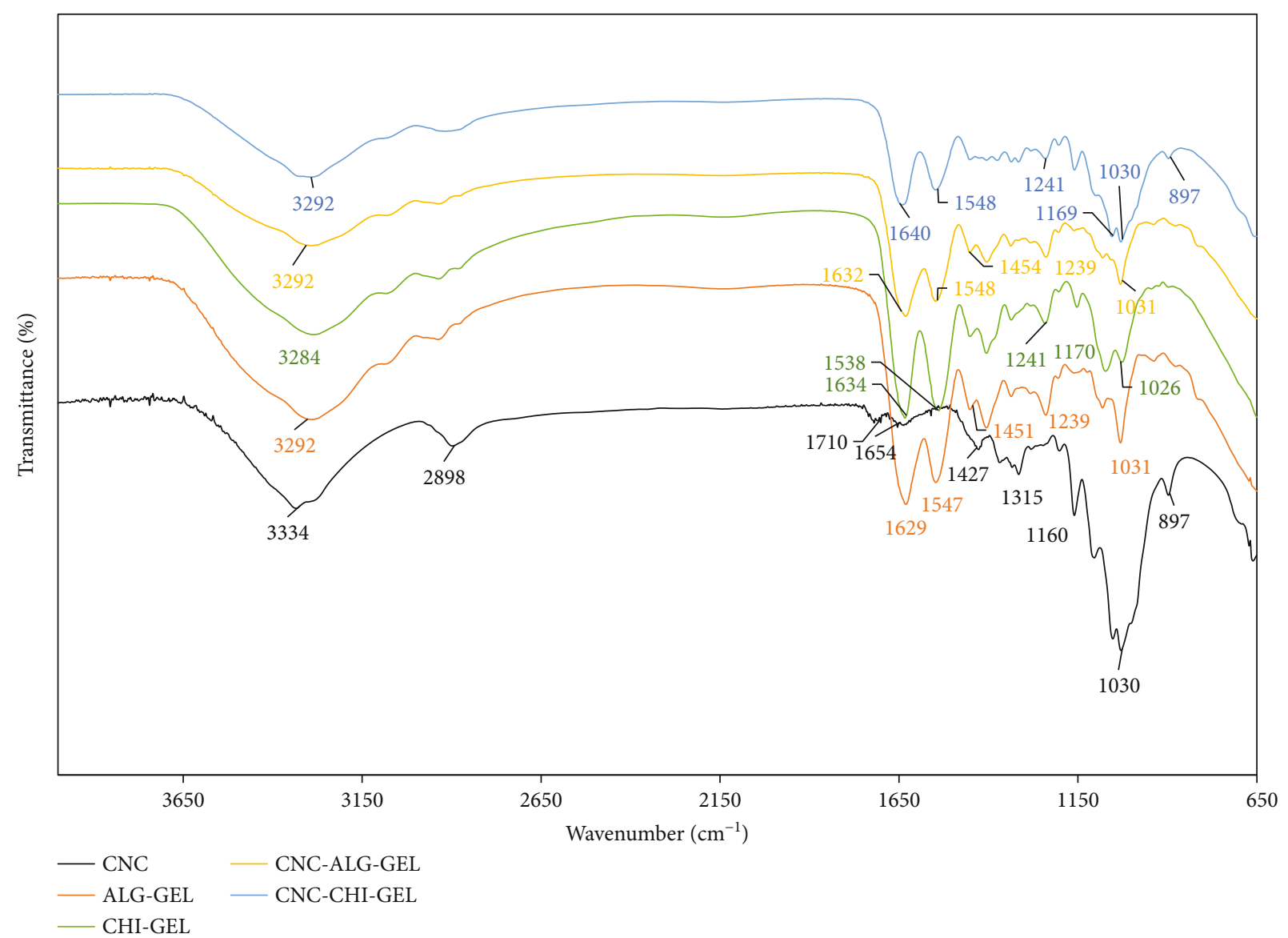

FIgURE 2: FT-IR spectra of CNC and hydrogels.

absorption bands at wave numbers at $3334 \mathrm{~cm}^{-1}, 2898 \mathrm{~cm}^{-1}$, $1654 \mathrm{~cm}^{-1}, 1427 \mathrm{~cm}^{-1}, 1315 \mathrm{~cm}^{-1}, 1150 \mathrm{~cm}^{-1}, 1030 \mathrm{~cm}^{-1}$, and $897 \mathrm{~cm}^{-1}$, which can be attributed to $\mathrm{O}-\mathrm{H}, \mathrm{C}-\mathrm{H}, \mathrm{COO}^{-}, \mathrm{O}-$ $\mathrm{H}$ bending, $\mathrm{C}-\mathrm{O}$ stretching, $\mathrm{CO}-\mathrm{O}-\mathrm{CO}$ stretching, and $\mathrm{C}-\mathrm{H}$ bending, respectively (Figure 2). The spectral data of $\mathrm{CNC}$ were in accordance with the spectral data of CNC extracted from rice husk [3]. The absorption band of CNC at a wave number at $1710 \mathrm{~cm}^{-1}$ can be attributed to stretching of carbonyl groups $(\mathrm{C}=\mathrm{O})$ resulting from the TEMPO reaction [22]. The characteristic absorption peaks of CNC-CHIGEL and CHI-GEL at wave number $\approx 1170 \mathrm{~cm}^{-1}$ can be assigned to the free amine group $\left(-\mathrm{NH}_{2}\right)$ of the chitosan molecule, whereas on the other hand, the absorption peak of CNC-ALG-GEL and ALG-GEL wave number $\approx 1450 \mathrm{c}$ $\mathrm{m}^{-1}$ could be related to the carboxylic group $(-\mathrm{COOH})$ of alginate [5]. In line with these reports, our findings correlated well with the results of zeta potential analyses as reported earlier. The pattern of absorption bands of all the hydrogels was in accordance with the FT-IR spectra of fish gelatin [17]. It can be noted that the appearance of a broad absorption band at wave number $\approx 1625 \mathrm{~cm}^{-1}$ of all hydrogel samples is an indicator of the successful formation of a complex between carboxylic groups of $\mathrm{CNC}$ and amino groups of biopolymers. Furthermore, it is stated that the intensity of the peak at region $\sim 1625 \mathrm{~cm}^{-1}$ of all hydrogel samples represents the successful cross-linking of the gelatin chain with

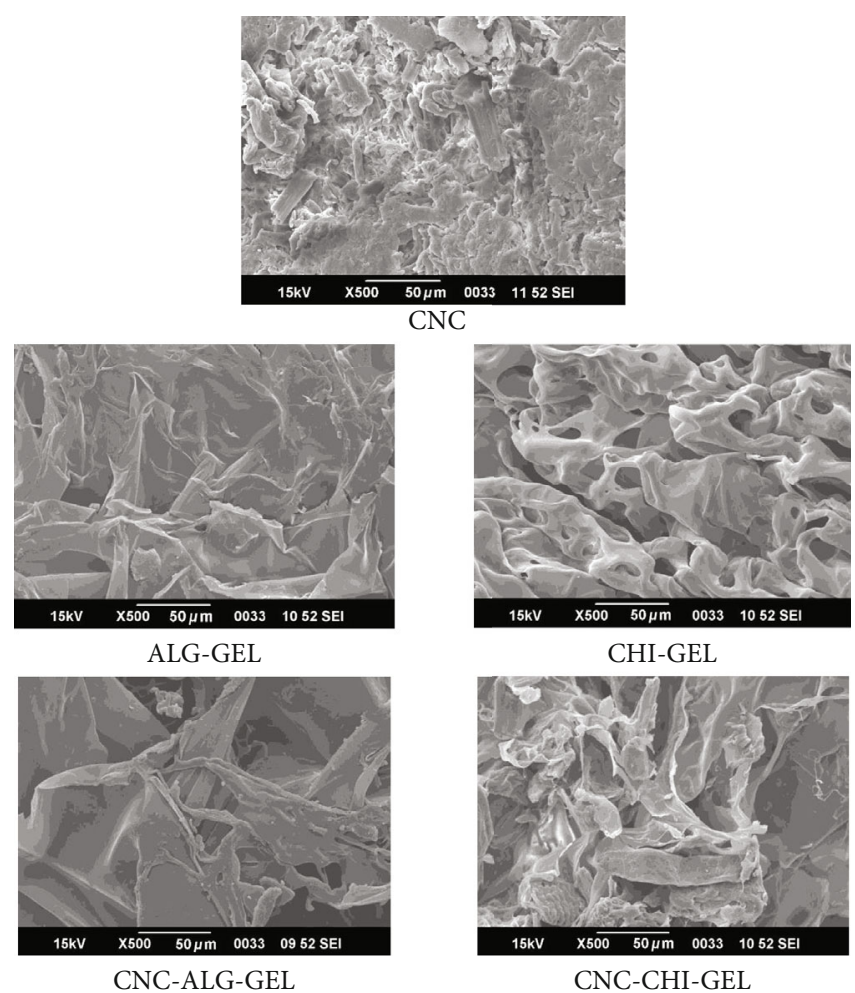

FIGURE 3: SEM of CNC and hydrogels. 


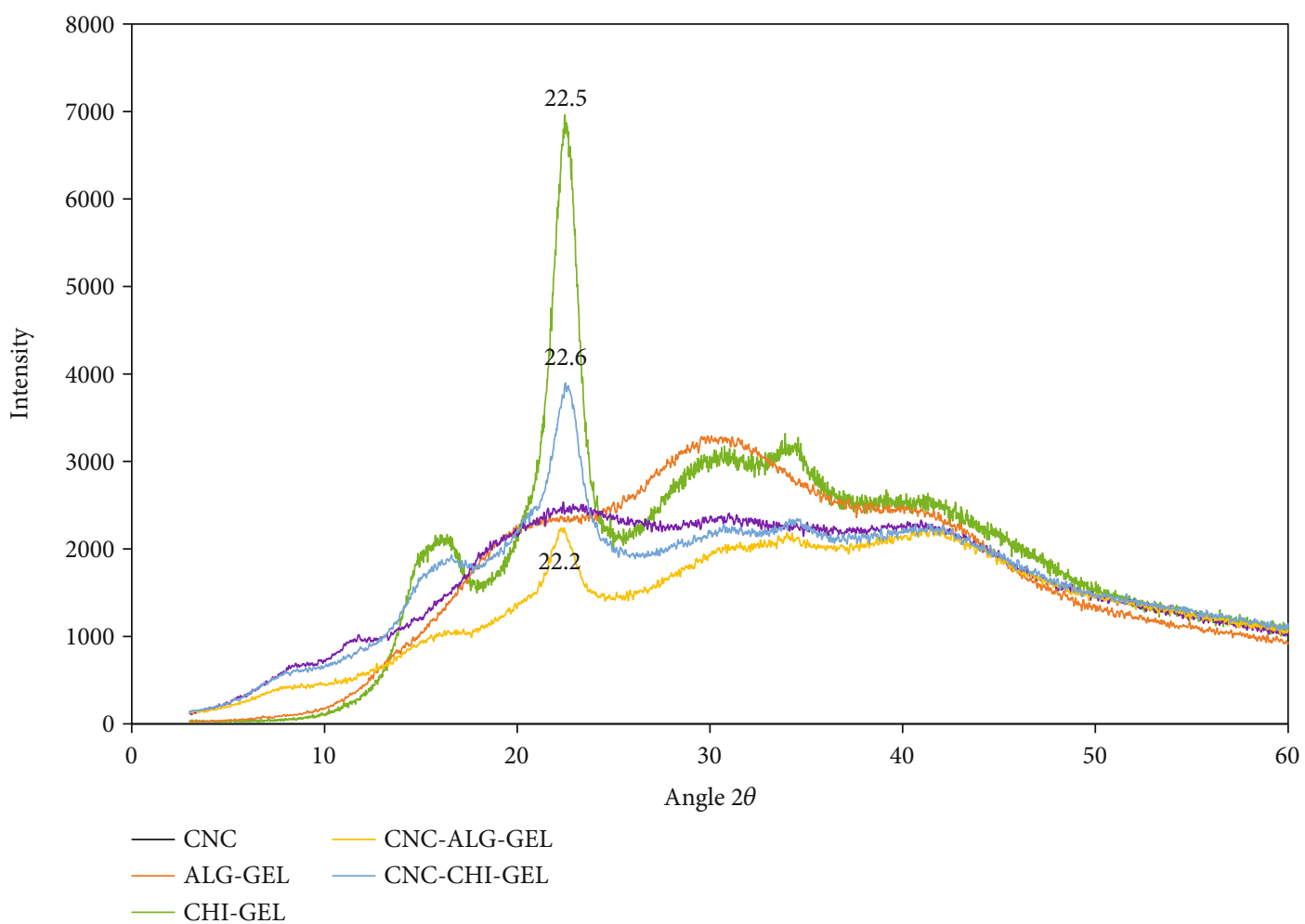

FIgURE 4: XRD of CNC and hydrogels.

the biopolymer hydrogels by overlapping the amide group of the gelatin molecule [3]. In another study by Wang et al., it was mentioned that the broad absorption peaks in the region $\left(3200 \mathrm{~cm}^{-1}-2900 \mathrm{~cm}^{-1}\right)$ of the spectrum of hydrogels illustrate the strong $\mathrm{O}-\mathrm{H}$ bond interaction between the biopolymer-gelatin backbone and CNC molecules [16]. The absorption bands of CNC at regions $\sim 1425 \mathrm{~cm}^{-1}$ and $\sim 1225 \mathrm{~cm}^{-1}$ were shifted to different frequencies in hydrogel samples after reinforcement, and this emphasizes the formation complex inter- and intrahydrogen bonding between CNC and polymer matrix [23]. The FT-IR results of the present study clearly indicated that the reinforcement of $\mathrm{CNC}$ has not altered the network structure of biopolymer molecules.

3.3. SEM Morphology. The morphology and microstructures of CNC and hydrogels were depicted in Figure 3. The morphology of CNC was found to have roughness with fractured surface. It is well known that the morphology of nanocrystals depends upon the severity of hydrolysis used for the reaction [4]. In the present study, the formic acid hydrolysis method was used for the preparation of CNC. The SEM micrographs of ALG-GEL, CNC-ALG-GEL, CHI-GEL, and CNC-CHI-GEL appeared to be smooth and also showed porous structures compared to the micrograph of $\mathrm{CNC}$, which indicated the blend miscibility, high homogeneity, and in situ dissolution of biopolymers to form blend mixtures [22]. The impact of smoothness and porosity was high in CNC-ALG-GEL and CNC-CHI-GEL hydrogels as compared to ALG-GEL and CHI-GEL. It is noticed that certain factors such as hydrogen bonding and complexity of CNC with biopolymers define the network structure formation of hydrogel membranes [24]. The uniform distribution of pores in the CNC-ALG-GEL and CNC-CHI-GEL hydrogels reflected the embedded nature of $\mathrm{CNC}$ which resulted from strong interfacial adhesion between the CNC and the biopolymer matrix. Technically, the roughness and smoothness of hydrogel membranes depend upon the concentration of CNC added to hydrogels [9]. In the present study, the interpenetrated networks in the hydrogel membranes due to the reinforcement of CNC were a good indication of superior mechanical properties and good stability against degradation, which correlated well with an earlier report [16]. Moreover, the hydrogels with these characteristics also support other features such as easy injectability and costeffectiveness which are more suitable for tissue engineering and regenerative medicine applications [1].

3.4. X-Ray Diffraction (XRD) Pattern. As shown in Figure 4, the CNC showed three cellulose characteristic peaks at $2 \theta$ $=14.2^{\circ}, 16.4^{\circ}$, and $22.5^{\circ}$, where the prominent characteristic sharp peak at $2 \theta=22.5^{\circ}$ attributing to (200) plane reflection of crystal lattice of alpha-cellulose and hemicelluloses [25]. The peak at $2 \theta=20^{\circ}$ of CHI-GEL indicates the semiamorphous nature of chitosan, whereas the peak at $2 \theta=19.9^{\circ}$ of ALG-GEL indicates the amorphous and crystalline nature of alginate $[5,18]$. The characteristic peak of $\mathrm{CNC}$ at $2 \theta=$ $22^{\circ}$ appeared in the CNC-ALG-GEL and CNC-CHI-GEL which indicated the presence of CNC in the hydrogel membrane matrix. However, this characteristic peak was slightly shifted, and the disparity in crystallinity can be due to intermolecular interactions. It was noticed that the intensity of 


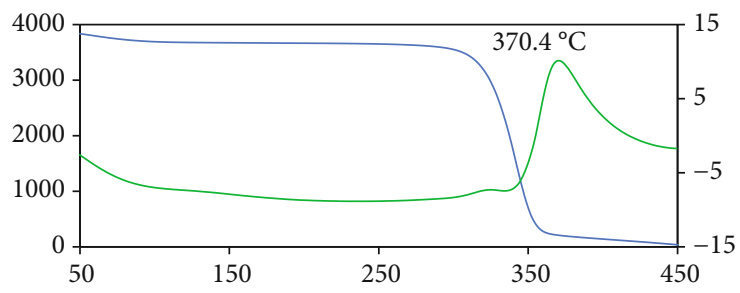

(A)

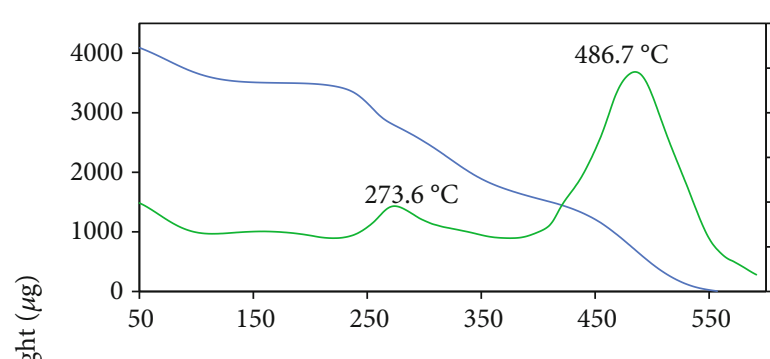

(B)

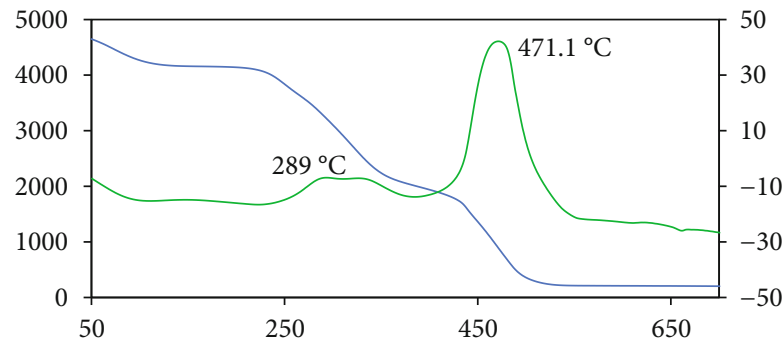

(D)
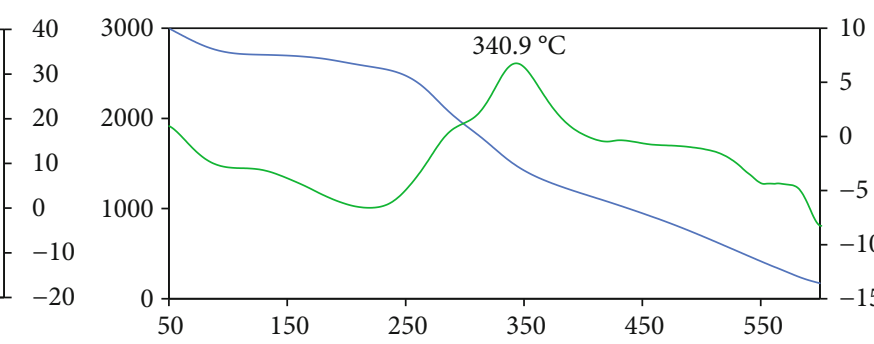

(C)

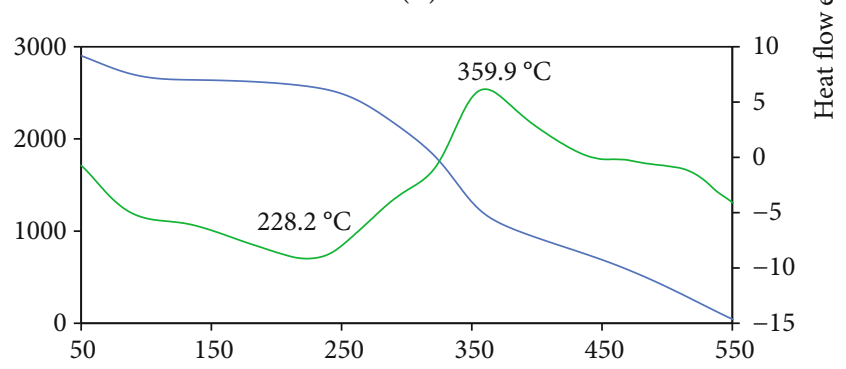

(E)

Temperature $\left({ }^{\circ} \mathrm{C}\right)$

(a)

Figure 5: Continued. 


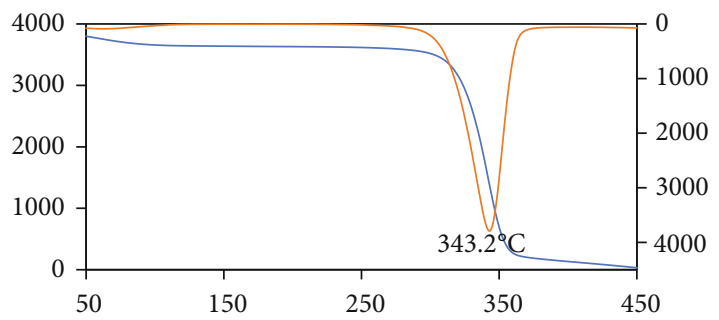

(A)

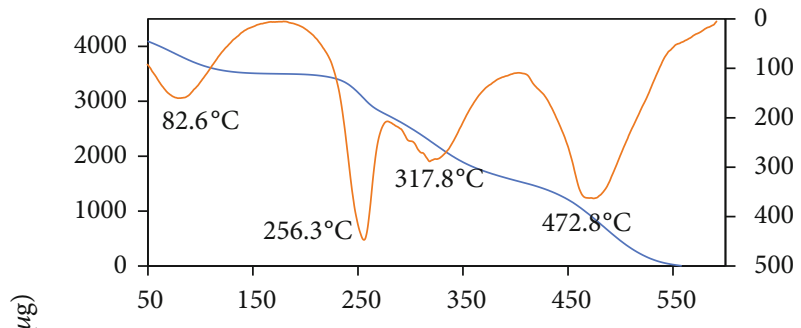

(B)
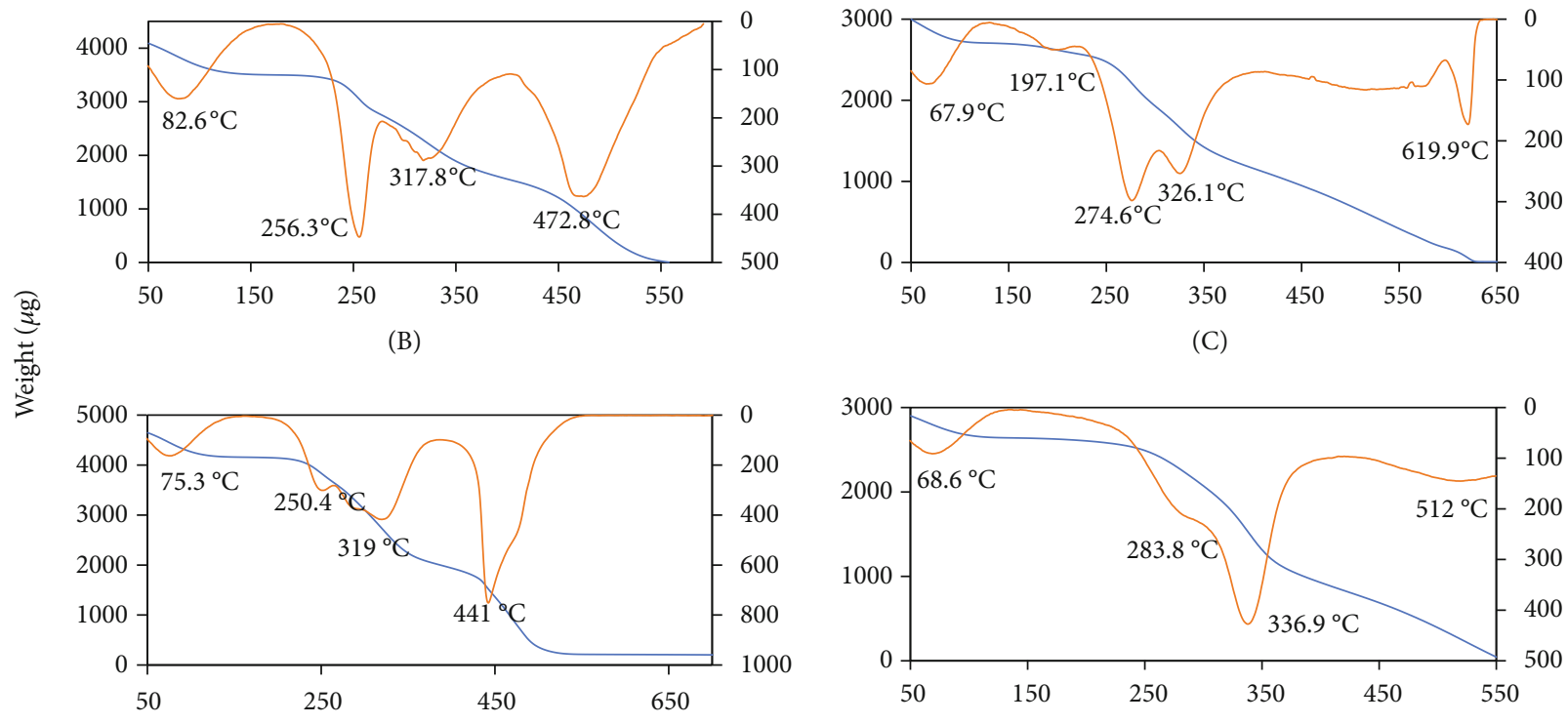

(C)

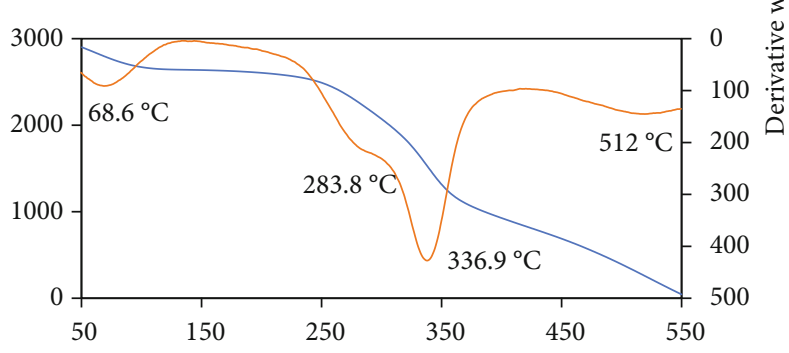

(D)

Temperature $\left({ }^{\circ} \mathrm{C}\right)$

(E)

(b)

Figure 5: (a) DTA curves of hydrogels (A) CNC, (B) ALG-GEL, (C) CHI-GEL, (D) CNC-ALG-GEL, and (E) CNC-CHI-GEL at 20 ${ }^{\circ}$ C/min heating rate. (b) DTG curves of hydrogels (A) CNC, (B) ALG-GEL, (C) CHI-GEL, (D) CNC-ALG-GEL, and (E) CNC-CHI-GEL at $20^{\circ} \mathrm{C} / \mathrm{min}$ heating rate.

the peak at $2 \theta=22^{\circ}$ of CNC-ALG-GEL was lesser as compared to that of CNC-CHI-GEL, which could be due to the difference in the percolation phenomenon of CNC in contact with alginate [8]. The intensity of the peak at $2 \theta=22^{\circ}$ of CNC-ALG-GEL was found to be increased as compared to that of CNC-CHI-GEL, due to the transcrystallization effect of nanocrystals. Furthermore, it can also be stated that the interaction between the CNC and the biopolymer matrix was one of the probable reasons for the increment of crystallinity [9]. However, XRD data in the present study revealed that the reinforcement of $\mathrm{CNC}$ does not change the structural uniformity of the hydrogel-based biopolymer matrix by improving the molecular ordering and mechanical properties as well as the amorphous nature of the polymer matrix.

3.5. Thermal Characteristics. DTG/DTA curves of hydrogel samples at $20^{\circ} \mathrm{C} / \mathrm{min}$ heating rate under inert atmosphere are depicted in Figures 5(a) and 5(b). The thermal characteristic data illustrated that all the hydrogel samples have shown three major degradation steps. Thermal degradation of polymers includes dehydration followed by decomposition along with breakage of intra- and interlinkages in the structure of polymers [26]. The first degradation step of $\mathrm{CNC}$ was shown between $50^{\circ} \mathrm{C}$ and $300^{\circ} \mathrm{C}$, which might be due to the loss of water bound to the polymer. The second degradation step was in the range of $300-360^{\circ} \mathrm{C}$, and the maximum degradation temperature at this point was found to be $343^{\circ} \mathrm{C}$. The final and third degradation step was found to be approximately at $450^{\circ} \mathrm{C}$. The first degradation steps of hydrogels ALG-GEL, CHI-GEL, CNC-ALG-GEL, and CNCCHI-GEL were found to be $82^{\circ} \mathrm{C}, 67^{\circ} \mathrm{C}, 75^{\circ} \mathrm{C}$, and $68^{\circ} \mathrm{C}$, respectively. The maximum degradation temperatures of hydrogels during the second step were observed to be $256^{\circ} \mathrm{C}, 274^{\circ} \mathrm{C}, 319^{\circ} \mathrm{C}$ and $336^{\circ} \mathrm{C}$, respectively. The maximum degradation temperature of hydrogels hardly changes with the incorporation of CNC in the biopolymer matrix. It indicated that the CNC retained the thermal stability of hydrogels due to the strong interactions between $\mathrm{CNC}$ and biopolymers [27]. The significant weight loss of CHI-GEL and CNC-CHI-GEL hydrogels at the $200-400^{\circ} \mathrm{C}$ temperature range can be attributed to the depolymerization of chitosan 
through deacetylation and cleavage of glycosidic linkages via dehydration and deamination, whereas in the case of ALGGEL and CNC-ALG-GEL, it could be due to chain scission and ring opening reactions of alginate [9]. Maximum weight loss was observed to be in the second degradation step which could be attributed to degradation and dislocation of backbone linkages as well as functional groups of biopolymers [26]. The final and third degradation steps of hydrogels ALG-GEL and CHI-GEL were found to be at $472^{\circ} \mathrm{C}$ and $619^{\circ} \mathrm{C}$, respectively, whereas for CNC-ALG-GEL and CNC$\mathrm{CHI}-\mathrm{GEL}$ hydrogels, they were at $441^{\circ} \mathrm{C}$ and $512^{\circ} \mathrm{C}$, respectively. It can be observed that the reinforcement of CNC to the hydrogels has reduced the degradation temperature. This might be due to the decomposition of CNC derivatives that indicated the carbonization of polymeric biomaterial [28]. This difference in the degradation temperature and weight loss between the hydrogels might be due to the difference in the formation of hydrogen bonding between the $\mathrm{CNC}$ and molecules of biopolymers, and cross-linkers [29].

Figures 5(a) and 5(b) and Table 2 illustrate the $T_{\mathrm{o}}$ (onset temperature), $T_{\mathrm{m}}$ (melting temperature), and $\Delta H$ (enthalpy) of hydrogels. $T_{\mathrm{o}}, T_{\mathrm{m}}$, and $\Delta H$ (enthalpy) of polymeric blends usually represent the kinetic hindrances that take place during crystallization and melting. CNC showed a $T_{\mathrm{m}}$ value of $370^{\circ} \mathrm{C}$ that corresponds to the decomposition of glycosyl units and the formation of carbonaceous residues [30]. The CNC-CHI-GEL hydrogels reinforced with CNC were found to have a high $T_{\mathrm{m}}$ value compared to that of CHI-GEL, whereas CNC-ALG-GEL was marginally lesser than ALG-GEL. In a previous study by Rescignano et al., it was noted that the $T_{\mathrm{m}}$ values of chitosan and alginate biomaterials incorporated with CNC were found to be $289^{\circ} \mathrm{C}$ and $317^{\circ} \mathrm{C}$, respectively [20]. In the present study, the $T_{\mathrm{m}}$ values of chitosan and alginate hydrogels reinforced with $\mathrm{CNC}$ were observed to be $359^{\circ} \mathrm{C}$ and $417^{\circ} \mathrm{C}$, respectively. The disparity in $T_{\mathrm{m}}$ values might be due to the formation of intermolecular hydrogen interactions between chitosan, alginate, and $\mathrm{CNC}$ at elevated temperature. As the $T_{\mathrm{m}}$ value goes beyond the elevated temperature, the biopolymer gets the tendency to donate hydrogen bonds and results in the production of more - $\mathrm{OH}$ groups, and these groups facilitate more interaction and influence the melting temperature of biopolymeric materials [8]. Moreover, chemically modified CNC incorporated in biopolymers influence the $T_{\mathrm{m}}$, due to strong interactions between chemically modified CNC and polymer matrices which could be originated from $T_{\mathrm{m}}$ difference [6]. Notably, the chemically modified CNC do not have any impact on the $T_{\mathrm{o}}$ of biopolymers [1]. The other resultant exothermic peaks of hydrogel membranes might be due to the decomposition of alginate as well as the breakdown of intermolecular structure of gelatin [31]. It is demonstrated from the present data that the biomaterial-based hydrogels reinforced with cellulose nanocrystals showed better thermal stability and miscibility as revealed by thermal gravity analysis.

3.6. Rheological Study. Different sorts of physicodynamic or computer rheological models such as Herschel-Bulkley, Newtonian, Bingham, Casson, and Power law were usually
TABLE 2: Thermal characteristics of CNC and hydrogels.

\begin{tabular}{lccc}
\hline Samples & $T_{\mathrm{o}}\left({ }^{\circ} \mathrm{C}\right)$ & $T_{\mathrm{m}}\left({ }^{\circ} \mathrm{C}\right)$ & $\Delta H(\mathrm{~mJ} / \mathrm{mg})$ \\
\hline CNC & $313.2 \pm 14.86$ & $370.4 \pm 16.82$ & $-618 \pm 23$ \\
ALG-GEL & $230.4 \pm 11.24$ & $486.7 \pm 17.80$ & $-5675 \pm 209$ \\
CHI-GEL & $237.7 \pm 12.45$ & $340.9 \pm 29.87$ & $-889 \pm 236$ \\
CNC-ALG-GEL & $237.8 \pm 13.56$ & $471.1 \pm 14.30$ & $-4992 \pm 215$ \\
CNC-CHI-GEL & $246.9 \pm 10.41$ & $359.9 \pm 13.74$ & $-707 \pm 55$ \\
\hline
\end{tabular}

TABLE 3: Herschel-Bulkley parameters for hydrogels.

\begin{tabular}{lcccc}
\hline Hydrogels & $T_{\mathrm{o}}$ & $k$ & $\eta^{\prime}$ & $R^{2}$ \\
\hline CNC & 0.03 & 0.74 & 1.38 & 0.99 \\
ALG-GEL & 0.14 & 1.40 & 1.04 & 0.99 \\
CNC-ALG-GEL & 0.04 & 0.96 & 1.26 & 0.99 \\
CHI-GEL & 0.08 & 20.5 & 0.80 & 0.99 \\
CNC-CHI-GEL & 0.06 & 11.7 & 1.06 & 0.99 \\
\hline
\end{tabular}

$T_{\mathrm{o}}$ : yield stress; $k$ : consistency coefficient; $\eta$ : flow behavior index; $R^{2}$ : regression coefficient.

used to present the flow profile of blends and hydrogels to describe shear stress and shear behaviour. The HerschelBulkley rheological model is mostly preferred due to its accuracy. In the present study, the rheological HerschelBulkley model was used to analyse the experimental result data (shear stress and viscosity-shear rate) of hydrogel solutions (Table 3$)$. The yield stress $\left(T_{\mathrm{o}}\right)$, flow behaviour index $(\eta$ ), and consistency coefficient $(k)$ were also calculated using the software provided with the rheometer. The yield stress $\left(T_{\mathrm{o}}\right)$ represents the interaction potential of colloidal particles in the biopolymer and also plays an important role in demonstrating the macrolattice structure of biopolymers [32]. The rheological Herschel-Bulkley model exhibited yield stress values for hydrogel samples which were studied (Table 3 ). The $T_{\mathrm{o}}$ and consistency coefficient $(k)$ values of ALG-GEL and CHI-GEL were found to have higher values as compared to those of CNC-ALG-GEL and CNC-CHIGEL hydrogels, respectively. It has been stated that the method of analysis and experimental condition influences the yield stress [33]. Several research studies have conflated views on the yield stress which could be linked with the transition between liquid and solid states and/or two same fluids with different viscosities [34].

The ALG-GEL exhibited shear thickening and Newtonian behaviour, whereas the CHI-GEL exhibited shear thinning and pseudoplastic behaviour, which represents an irreversible structural breakdown (Figure 6). The Newtonian behaviour of ALG-GEL at low shear rates was characterized by shear rate-independent viscosity. The shear thinning behaviour of CHI-GEL indicates the disrupted intermolecular junctions with a slower rate of reformations, which tends to drop in viscosity at a lower shear rate [35]. As the shear rate increases, the viscosity of CNC-ALG-GEL and CNCCHI-GEL hydrogels tends to decrease. This might be due to the distributed and weak nature of the hydrogel network, 


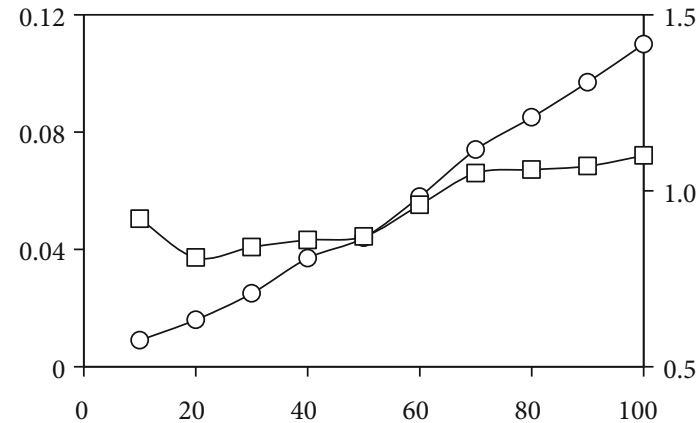

(a)

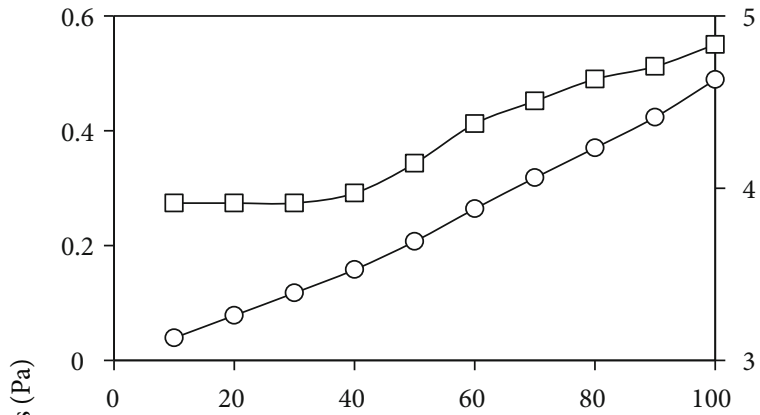

(b)

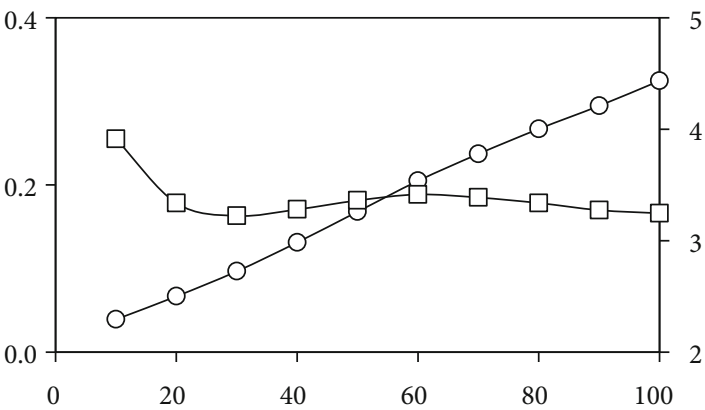

(d)

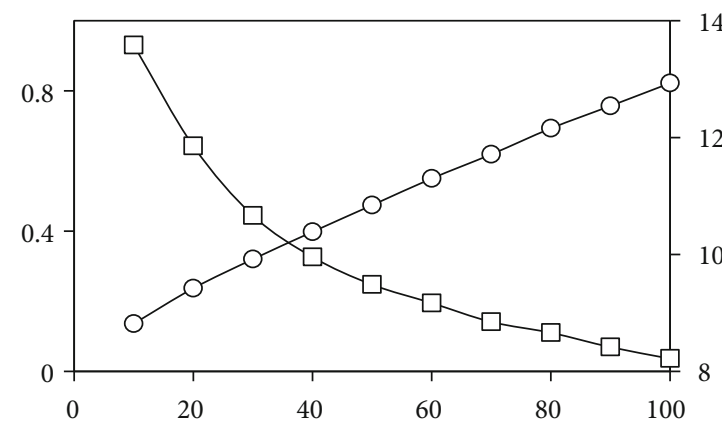

(c)

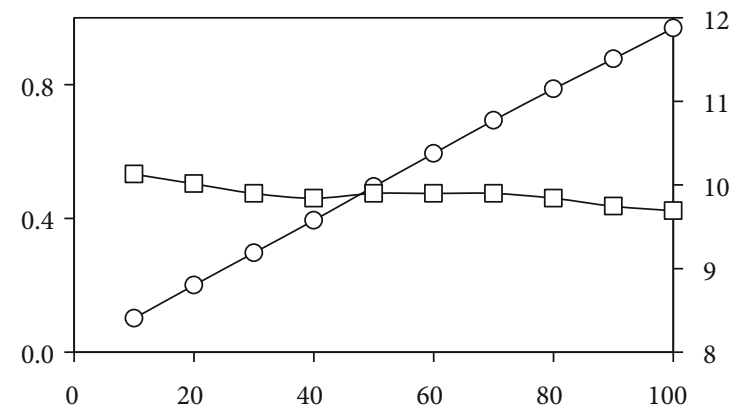

(e)

Shear rate $(1 / S)$

FIGURE 6: Flow properties (shear stress vs. shear rate and viscosity vs. shear rate) of hydrogels. (a) CNC, (b) ALG-GEL, (c) CHI-GEL, (d) CNC-ALG-GEL, and (e)CNC-CHI-GEL.

and this leads to release of the entrapped liquid which resists the flow behaviour, thus inducing the lower viscosity [36]. This nonlinear relationship represents the higher viscosity of hydrogels which is a strong indication on the impact of cross-linking [22]. The viscosity of a shear thinning liquid has an inversely proportional relationship with the shear rate, whereas a shear thickening liquid has a directly proportional relationship between viscosity and the shear rate [37]. Pseudoplastic nature is important for topical formulations that have viscous behaviour under static conditions; nevertheless, this viscous behaviour tends to decrease at a higher shear rate which results in better spreadability and improves the drug permeation of specific substances upon topical application [11]. By the reinforcement of CNC to hydrogels, the viscosity vs. shear rate curve of CNC-ALG-GEL and CNC-CHI-GEL hydrogels has transformed to a plateau shape, due to the breaking down of CNC domains by shearing force [38]. With regard to the flow behaviour index, $\eta$ $=1$ for a Newtonian fluid and $\eta<1$ for a shear thinning fluid or rheofluid, whereas $\eta>1$ for shear thickening or rheothickening fluid. In the present study, $\eta$ values of CNC-ALG-GEL and CNC-CHI-GEL hydrogels indicated a Newtonian and shear thickening nature. This phenomenon of transition represents the network structure formed by $\mathrm{CNC}$ within alginate, chitosan, and gelatin which was constructed by strong hydrogen interactions and eventually maintains the viscosity $[39,40]$. However, the increase in $\eta$ values of CNC-ALG-GEL and CNC-CHI-GEL hydrogels was also an indication of the shear thickening nature [2]. The biomaterial-based hydrogels reinforced with CNC showed a dependent behaviour which results from rheological characterization. 


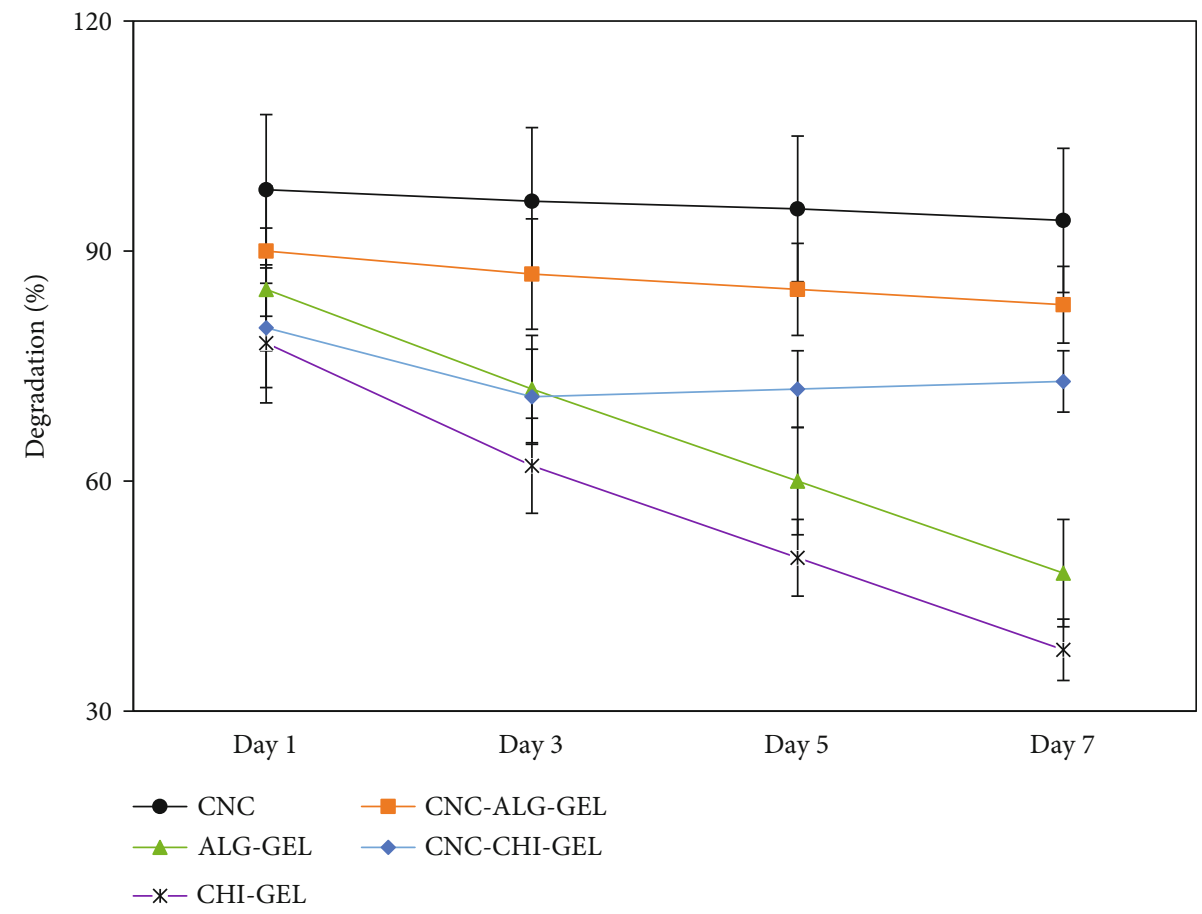

FIgURE 7: In vitro degradation of CNCs and hydrogels.

3.7. In Vitro Degradation. The ALG-GEL and CHI-GEL hydrogels were found to have $50 \%$ of weight loss from day 1 to day 7 (Figure 7). This illustrates that the majority of gelatin content in the respective hydrogel samples were susceptible to enzymatic degradation. In the hydrogel samples (CNC-ALG-GEL and CNC-CHI-GEL), 15\% of weight loss were significantly observed from day 1 to day 7 . In a study by Wang et al. [16], the hydrogels were prepared using alginate and gelatin biopolymers; moreover, the hydrogels without CNC were found to have 50\% weight loss, and the hydrogels with CNC have significantly improved resistance to biodegradation. This improved resistance to enzymatic degradation might be due to homogenous dispersion and hydrophobicity [16]. It was believed that the CNC might have helped in creating the interpenetrated matrix between biomaterials alginate, chitosan, and gelatin due to crosslinking and can be expected to distribute uniformly by hydrogen bonding and hydrophobic interactions. Wen et al. [41] developed interpenetrated network (IPN) hydrogels with $25 \%$ of weight loss observed within $2 \mathrm{~h}$ of enzymatic degradation. They stated that the biomaterials blended with gelatin tend to have superior mechanical characteristics which resulted from interpenetrated matrix, and this provides good stability against degradation [41]. Furthermore, the crystallinity and hydrophilicity are important factors that influence the degradation rate. In addition, the surface hydrophilicity tends to cause the invasion of the degradation medium and water into the amorphous regions of CNC [42]. In this viewpoint of degradation, the biomaterial-based hydrogels reinforced with $\mathrm{CNC}$ are mostly recommended for bone and tissue engineering applications.
3.8. Cytotoxicity. The in vitro cytotoxicity of $\mathrm{CNC}$ and hydrogel membranes was investigated using the $L 929$ cell line by MTT assay, and absorbance was read at $570 \mathrm{~nm}$ to evaluate cell viability [43]. In this method, the viability of the $L 929$ fibroblast cell line was observed after $24 \mathrm{~h}$ of incubation. According to GB/T 16886.5-2003 (ISO 109935:1999), the biomaterial-based hydrogel membranes with cell viability more than $75 \%$ can be considered as noncytotoxic and are recommended for biomedical applications. The cell viabilities of ALG-GEL and CHI-GEL hydrogels were significantly higher than those of CNC $(p<0.05)$. The CNC-ALG-GEL and CNC-CHI-GEL hydrogel membranes reinforced with $\mathrm{CNC}$ had $96 \%$ and $97 \%$ cell viabilities, respectively, which were significantly higher than $\mathrm{CNC}$, ALG-GEL, and CHI-GEL hydrogels $(p<0.05)$ (Figures 8(a) and 8(b)).

The alginate and chitosan bionanocomposite membranes reinforced with $\mathrm{CNC}$ enhanced the viability of NIH-3T3 fibroblasts $[16,24]$. In an another study by Shaheen et al., the chitosan-alginate-hydroxyapatite scaffolds fabricated with $\mathrm{CNC}$ were found to have $100 \%$ cell viability and promising cell growth and cell adherence [5]. In a previous study of our lab, promising biocompatibility characteristics of polyvinyl alcohol/starch polymer were proven [44]. It was noticed that the mechanical properties and zwitterion polymer network provide the suitable environment for cell growth and proliferation [23]. Interaction of viable cells with the substratum matrix of hydrogel membranes reinforced with CNC via physicobiochemical and molecular interactions and surface topological parameters has an essential role in the cytocompatibility of biomaterial membranes such as cell adhesion, differentiation, proliferation, and migration 


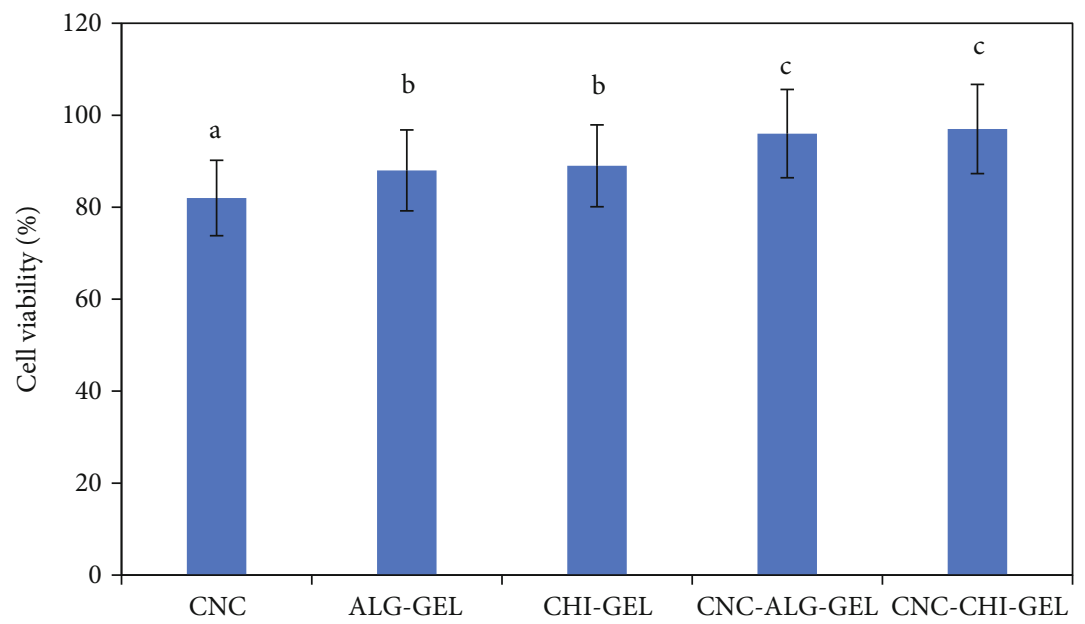

(a)

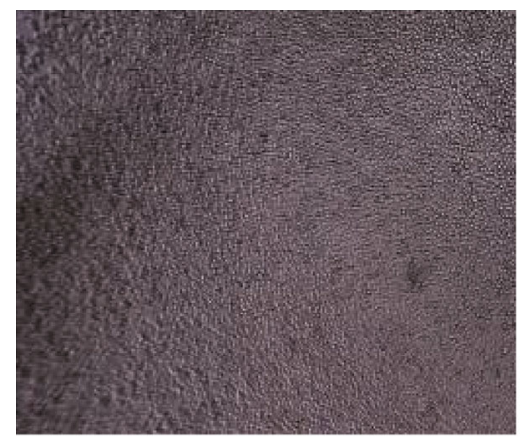

Untreated control

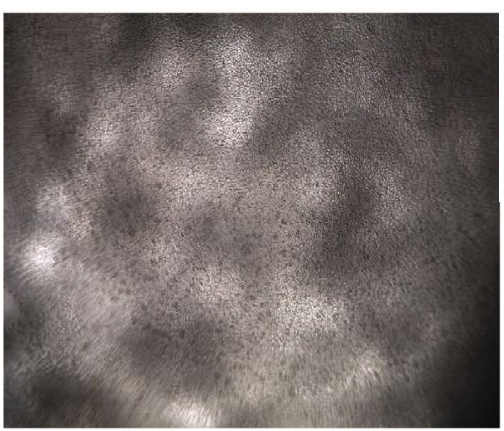

CHI-GEL

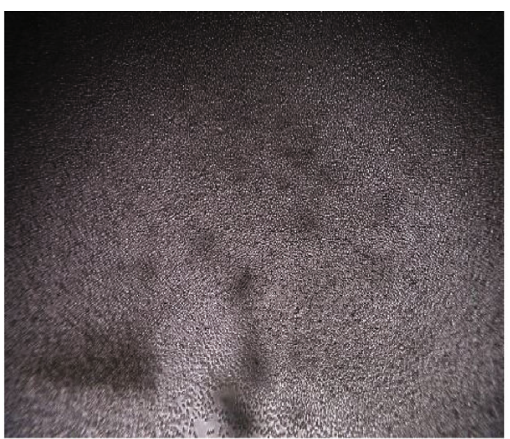

$\mathrm{CNC}$

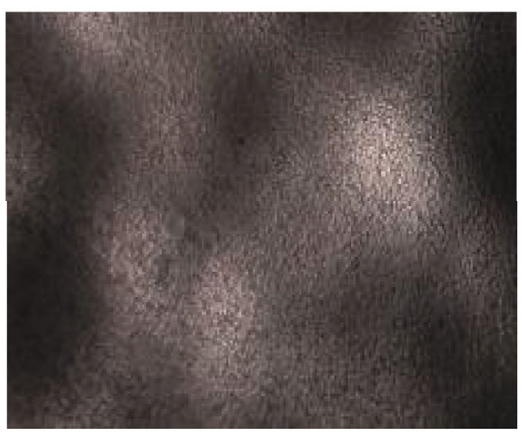

CNC-CHI-GEL

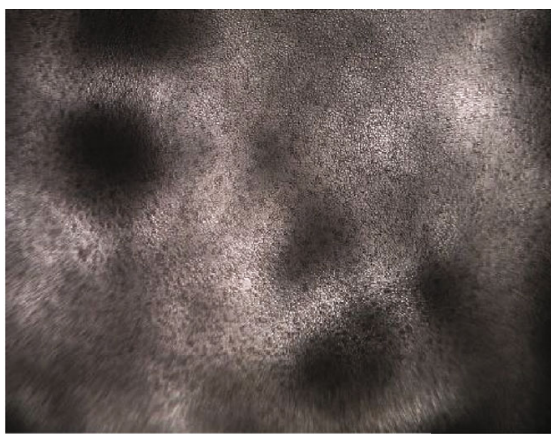

ALG-GEL

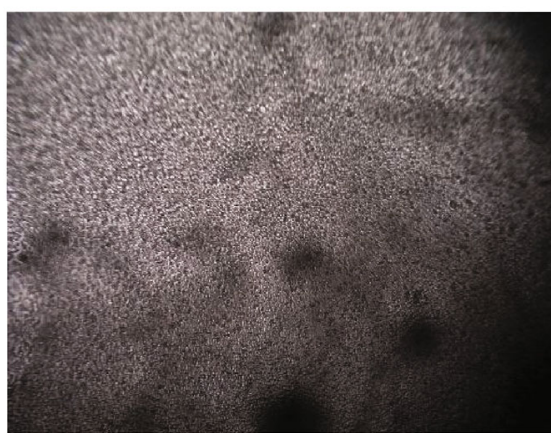

CNC-CHI-GEL

(b)

Figure 8: (a) Cell viability assay (different letters indicate significant difference; $p<0.05$ ). (b) Fluorescence microscope images of CNC and hydrogel membranes at $\times 10$ magnification.

[5]. Furthermore, the pore size of hydrogel membranes is mostly controlled by the zeta potential which enables the transportation of necessary bioactive molecules and migration of cells within the matrix of hydrogel membranes. In the present study, the zeta potential values of CNC-ALGGEL, and CNC-CHI-GEL were quite different from those of ALG-GEL and CHI-GEL which might be due to the reinforcement of CNC. Surface charge modification by TEMPO was also most likely a probable reason for changes in zeta potential values. It was noted from a previous study that the CNC and gelatin promote the cell growth by forming mimetics of native bone tissue [45].

\section{Conclusion}

In the present study, biopolymer-based hydrogels reinforced with CNC were synthesized. Electrostatic chemical attractions, cross-linking of zinc ions, and intermolecular interactions were involved between CNC and alginate, chitosan, and gelatin used for the hydrogel preparation. These 
biopolymer-based hydrogel membranes were characterized by desirable physicochemical and morphological properties. Surface charge modification and the reinforcement of CNC enhanced the crystallinity and stability against degradation. Cytotoxicity studies carried out with L929 cell lines have confirmed its biocompatible nature for applications in tissue engineering, drug delivery, and biomedical fields. Also, hydrogels reinforced with CNC enabled effective cell adhesion, growth, and proliferation as evidenced in our study. However, further experimental studies need to be carried out for the applicability of these hydrogel membranes reinforced with $\mathrm{CNC}$ as a supportive biomaterial membrane in the treatment of burn wounds, skin implantations, in vivo drug delivery, and tissue transplantation.

\section{Data Availability}

Sufficient data have been included in the manuscript. Additional data can be kindly requested from the authors.

\section{Conflicts of Interest}

There is no conflict of interest to declare.

\section{Acknowledgments}

The authors would like to express their sincere gratitude to ICAR for providing funds to carry out the research work under the ICAR-National Fellow Scheme. The authors acknowledge the Director, ICAR-Central Institute of Fisheries Technology (ICAR-CIFT), Cochin, Kerala, India, for providing the facilities to carry out this work and also for granting permission to publish the data acquired from the study. The authors are grateful to Mrs. PA Jaya (Technical Officer), Mr. P. Suresh (Senior Technician), and Mrs. N. Lekha (Technical Officer) ICAR-Central Institute of Fisheries Technology (CIFT), Cochin, Kerala, for providing technical support to carry out the analyses.

\section{References}

[1] Y. Habibi, L. A. Lucia, and O. J. Rojas, "Cellulose nanocrystals: chemistry, self-assembly, and applications," Chemical Reviews, vol. 110, no. 6, pp. 3479-3500, 2010.

[2] S. D. Dutta, J. Hexiu, D. K. Patel, K. Ganguly, and K. T. Lim, "3D-printed bioactive and biodegradable hydrogel scaffolds of alginate/gelatin/cellulose nanocrystals for tissue engineering," International Journal of Biological Macromolecules, vol. 167, pp. 644-658, 2021.

[3] S. Y. Ooi, I. Ahmad, and M. C. I. M. Amin, "Cellulose nanocrystals extracted from rice husks as a reinforcing material in gelatin hydrogels for use in controlled drug delivery systems," Industrial Crops and Products, vol. 93, pp. 227-234, 2016.

[4] H. du, C. Liu, X. Mu et al., "Preparation and characterization of thermally stable cellulose nanocrystals via a sustainable approach of $\mathrm{FeCl}_{3}$-catalyzed formic acid hydrolysis," Cellulose, vol. 23, no. 4, pp. 2389-2407, 2016.

[5] T. I. Shaheen, A. S. Montaser, and S. Li, "Effect of cellulose nanocrystals on scaffolds comprising chitosan, alginate and hydroxyapatite for bone tissue engineering," International
Journal of Biological Macromolecules, vol. 121, pp. 814-821, 2019.

[6] S. P. Akhlaghi, R. C. Berry, and K. C. Tam, "Surface modification of cellulose nanocrystal with chitosan oligosaccharide for drug delivery applications," Cellulose, vol. 20, no. 4, pp. 17471764, 2013.

[7] Z. Hosseinidoust, M. N. Alam, G. Sim, N. Tufenkji, and T. G. van de Ven, "Cellulose nanocrystals with tunable surface charge for nanomedicine," Nanoscale, vol. 7, no. 40, pp. 16647-16657, 2015.

[8] T. Huq, S. Salmieri, A. Khan et al., "Nanocrystalline cellulose (NCC) reinforced alginate based biodegradable nanocomposite film," Carbohydrate Polymers, vol. 90, no. 4, pp. 17571763, 2012.

[9] H. Celebi and A. Kurt, "Effects of processing on the properties of chitosan/cellulose nanocrystal films," Carbohydrate Polymers, vol. 133, pp. 284-293, 2015.

[10] N. Lin, A. Gèze, D. Wouessidjewe, J. Huang, and A. Dufresne, "Biocompatible double-membrane hydrogels from cationic cellulose nanocrystals and anionic alginate as complexing drugs codelivery," ACS Applied Materials \& Interfaces, vol. 8, no. 11, pp. 6880-6889, 2016.

[11] L. Esposito, A. I. Barbosa, T. Moniz et al., "Design and characterization of sodium alginate and poly(vinyl) alcohol hydrogels for enhanced skin delivery of quercetin," Pharmaceutics, vol. 12, no. 12, p. 1149, 2020.

[12] M. Karzar Jeddi and M. Mahkam, "Magnetic nano carboxymethyl cellulose-alginate/chitosan hydrogel beads as biodegradable devices for controlled drug delivery," International Journal of Biological Macromolecules, vol. 135, pp. 829-838, 2019.

[13] G. M. A. Ndong Ntoutoume, R. Granet, J. P. Mbakidi et al., "Development of curcumin-cyclodextrin/cellulose nanocrystals complexes: new anticancer drug delivery systems," Bioorganic \& Medicinal Chemistry Letters, vol. 26, no. 3, pp. 941945, 2016.

[14] P. Bertsch, L. Schneider, G. Bovone, M. W. Tibbitt, P. Fischer, and S. Gstöhl, "Injectable biocompatible hydrogels from cellulose nanocrystals for locally targeted sustained drug release," ACS Applied Materials \& Interfaces, vol. 11, no. 42, pp. 38578-38585, 2019.

[15] M. Åhlén, G. K. Tummala, and A. Mihranyan, "Nanoparticleloaded hydrogels as a pathway for enzyme-triggered drug release in ophthalmic applications," International Journal of Pharmaceutics, vol. 536, no. 1, pp. 73-81, 2018.

[16] K. Wang, K. C. Nune, and R. D. K. Misra, "The functional response of alginate-gelatin-nanocrystalline cellulose injectable hydrogels toward delivery of cells and bioactive molecules," Acta Biomaterialia, vol. 36, pp. 143-151, 2016.

[17] P. K. Dara, M. Raghavankutty, N. Sebastian et al., "Rheological, physico-chemical, and surface-active properties of gelatin extracted from bigeye tuna (Thunnus obesus) skin waste," Journal of Aquatic Food Product Technology, vol. 29, no. 5, pp. 428-444, 2020.

[18] P. K. Dara, M. Raghavankutty, G. Balaraman et al., "Biocompatibility and histopathological evaluation of chitosan nanoparticles grafted fish gelatin bio-nanocomposite membranes in rats," Iranian Polymer Journal, vol. 30, no. 9, pp. 953-964, 2021.

[19] B. Li, W. Xu, D. Kronlund et al., "Cellulose nanocrystals prepared via formic acid hydrolysis followed by TEMPO- 
mediated oxidation," Carbohydrate Polymers, vol. 133, pp. 605-612, 2015.

[20] N. Rescignano, E. Fortunati, I. Armentano et al., "Use of alginate, chitosan and cellulose nanocrystals as emulsion stabilizers in the synthesis of biodegradable polymeric nanoparticles," Journal of Colloid and Interface Science, vol. 445, pp. 31-39, 2015.

[21] Y. Hu, X. Jiang, Y. Ding, H. Ge, Y. Yuan, and C. Yang, "Synthesis and characterization of chitosan-poly(acrylic acid) nanoparticles," Biomaterials, vol. 23, no. 15, pp. 3193-3201, 2002.

[22] R. Abouzeid, R. Khiari, D. Beneventi, and A. Dufresne, "Biomimetic mineralization of 3D printed alginate/TEMPO-oxidized cellulose nanofibril scaffolds," in abstracts of papers of the American Chemical Society, vol. 257, American Chemical Society, NW, WASHINGTON DC, USA, 2019.

[23] N. Chiaoprakobkij, S. Seetabhawang, N. Sanchavanakit, and M. Phisalaphong, "Fabrication and characterization of novel bacterial cellulose/alginate/gelatin biocomposite film," Journal of Biomaterials Science, Polymer Edition, vol. 30, no. 11, pp. 961-982, 2019.

[24] K. Madhusudana Rao, A. Kumar, and S. S. Han, "Polysaccharide based bionanocomposite hydrogels reinforced with cellulose nanocrystals: drug release and biocompatibility analyses," International Journal of Biological Macromolecules, vol. 101, pp. 165-171, 2017.

[25] P. Lu and Y. L. Hsieh, "Preparation and properties of cellulose nanocrystals: rods, spheres, and network," Carbohydrate Polymers, vol. 82, no. 2, pp. 329-336, 2010.

[26] S. Lopes, L. Bueno, F. D. Aguiar Júnior, and C. Finkler, "Preparation and characterization of alginate and gelatin microcapsules containing Lactobacillus rhamnosus," Anais da Academia Brasileira de Ciências, vol. 89, no. 3, pp. 16011613, 2017.

[27] Q. Li, J. Zhou, and L. Zhang, "Structure and properties of the nanocomposite films of chitosan reinforced with cellulose whiskers," Journal of Polymer Science Part B: Polymer Physics, vol. 47, no. 11, pp. 1069-1077, 2009.

[28] C. F. Bellani, E. Pollet, A. Hebraud et al., "Morphological, thermal, and mechanical properties of poly( $\varepsilon$-caprolactone)/poly( $\varepsilon$-caprolactone)-grafted-cellulose nanocrystals mats produced by electrospinning," Journal of Applied Polymer Science, vol. 133, no. 21, 2016.

[29] S. Parvez, M. M. Rahman, M. A. Khan et al., "Preparation and characterization of artificial skin using chitosan and gelatin composites for potential biomedical application," Polymer Bulletin, vol. 69, no. 6, pp. 715-731, 2012.

[30] S. S. Hindi, "Nanocrystalline cellulose: synthesis from pruning waste of Zizyphus spina christi and characterization," Nanoscience and Nanotechnology, vol. 4, no. 3, pp. 106-114, 2017.

[31] C. Xiao, H. Liu, Y. Lu, and L. Zhang, "Blend films from sodium alginate and gelatin solutions," Journal of Macromolecular Science, Part A, vol. 38, no. 3, pp. 317-328, 2001.

[32] V. Sharma, A. Jaishankar, Y. C. Wang, and G. H. McKinley, "Rheology of globular proteins: apparent yield stress, high shear rate viscosity and interfacial viscoelasticity of bovine serum albumin solutions," Soft Matter, vol. 7, no. 11, pp. 5150-5160, 2011.

[33] D. D. Christianson and E. B. Bagley, "Yield stresses in dispersions of swollen, deformable cornstarch granules," Cereal chemistry, vol. 61, no. 6, pp. 500-503, 1984.
[34] P. C. F. Møller, A. Fall, and D. Bonn, "Origin of apparent viscosity in yield stress fluids below yielding," Europhysics Letters, vol. 87, no. 3, article 38004, 2009.

[35] N. el Miri, K. Abdelouahdi, A. Barakat et al., "Bio-nanocomposite films reinforced with cellulose nanocrystals: rheology of film-forming solutions, transparency, water vapor barrier and tensile properties of films," Carbohydrate Polymers, vol. 129, pp. 156-167, 2015.

[36] B. V. Jyoti and S. W. Baek, "Rheological characterization of ethanolamine gel propellants," Journal of Energetic Materials, vol. 34, no. 3, pp. 260-278, 2016.

[37] Z. Liu, L. Zhang, A. Malfliet, B. Blanpain, and M. Guo, "NonNewtonian behavior of solid-bearing silicate melts: An experimental study," Journal of Non-Crystalline Solids, vol. 493, pp. 65-72, 2018.

[38] D. Liu, X. Chen, Y. Yue, M. Chen, and Q. Wu, "Structure and rheology of nanocrystalline cellulose," Carbohydrate Polymers, vol. 84, no. 1, pp. 316-322, 2011.

[39] C. Qiao, G. Chen, J. Zhang, and J. Yao, "Structure and rheological properties of cellulose nanocrystals suspension," Food Hydrocolloids, vol. 55, pp. 19-25, 2016.

[40] P. K. Dara, A. Geetha, U. Mohanty et al., "Extraction and characterization of myofibrillar proteins from different meat sources: a comparative study," Journal of Bioresources and Bioproducts, 2021.

[41] C. Wen, L. Lu, and X. Li, "Mechanically robust gelatin-alginate IPN hydrogels by a combination of enzymatic and ionic crosslinking approaches," Macromolecular Materials and Engineering, vol. 299, no. 4, pp. 504-513, 2014.

[42] M. Cheng, Z. Qin, S. Hu, S. Dong, Z. Ren, and H. Yu, “Achieving long-term sustained drug delivery for electrospun biopolyester nanofibrous membranes by introducing cellulose nanocrystals," ACS Biomaterials Science \& Engineering, vol. 3, no. 8, pp. 1666-1676, 2017.

[43] H. W. Sung, R. N. Huang, L. L. Huang, and C. C. Tsai, "In vitro evaluation of cytotoxicity of a naturally occurring crosslinking reagent for biological tissue fixation," Journal of Biomaterials Science, Polymer Edition, vol. 10, no. 1, pp. 63-78, 1999.

[44] P. K. Dara, M. Raghavankutty, G. K. Sivaraman et al., "Biomodulation of poly (vinyl alcohol)/starch polymers into composite-based hybridised films: physico-chemical, structural and biocompatibility characterization," Journal of Polymer Research, vol. 28, no. 7, pp. 1-12, 2021.

[45] J. M. Dugan, J. E. Gough, and S. J. Eichhorn, "Directing the morphology and differentiation of skeletal muscle cells using oriented cellulose nanowhiskers," Biomacromolecules, vol. 11, no. 9, pp. 2498-2504, 2010. 